\title{
Integrating aspects of cultural and environmental sensitivities into affordable housing in the Arab Gulf region
}

\author{
A. Mokhtar \\ School of Architecture and Design, American University of Sharjah, \\ United Arab Emirates
}

\begin{abstract}
Supported by strong oil-based economies, governments in several Gulf Arab states are committed to providing their citizens with free or highly subsidized housing. These are typically offered in the form of three or four bedroomed, single or double storey villas built on a freely provided piece of land. Thousands of these units are constructed every year to satisfy the growing population in these states. Unfortunately, the designs for most of these units follow ubiquitous design guidelines that do not fit well with a variety of cultural requirements for housing in the region. In addition, these designs are not sensitive with regards to the climate in which they are built. Consequently, both house owners and responsible government agents have begun to express their dissatisfaction with these designs. The lack of cultural sensitivity relating to the need for high levels of privacy has forced many owners to make costly modifications, and often limits their use and enjoyment of their property. The lack of environmental sensitivity has resulted in inflated government budgets that are needed to supply subsidized electricity and water to these houses. As a result, both owners and government agents now demand better designs, ones that are sensitive to the culture as well as the environment. This paper provides a different design approach that aims to satisfy these demands by integrating both cultural and environmental requirements. The paper investigates these requirements, shows a proposed architectural design, and critically reviews the design identifying its abilities and failures to satisfy and integrate these requirements.

Keywords: architectural sustainable design, cultural sensitivity, housing, Arab Gulf, courtyard houses.
\end{abstract}




\section{Introduction}

This paper aims to put forward for discussion a design approach that intends to integrate both environmental and cultural aspects of the architectural design for private houses. Such integration aspires to achieve sustainability not only in aspects such as energy and water but also extends to covering the sustainability of human cultures. The Arabian Gulf region was selected as the context for this discussion because of its unique culture that is being threatened by the very large percentage of expatriates (which can exceed 80 percent of the population), and the rapid change in lifestyle which includes, among other things, the expectations for private houses.

With strong economies supported by oil revenues, governments in these countries provide their less fortunate citizens with free economy housing. Thousands of such units are constructed annually in the region. Traditionally, the housing unit will be a villa with four to five bedrooms on a piece of land that measures about 800 square meters. While these specifications are considered luxurious in many parts of the world, they are seen as economical for citizens (natives) of the region due to large family sizes and high per capita incomes. Energy and water are typically provided at highly subsidized prices. Figure 1 shows a sample of the provided house.

However, several factors are now beginning to make such model of provision unsustainable. One factor is the rapid increase in the number of marriage-age citizens, which has enlarged the demand for such housing. The other important factor is the construction boom in the region. This has sharply inflated not only the cost of providing the houses, but more critically the cost of the infrastructure needed to provide subsidized water and energy. As these countries hardly have a system of taxation, the economic boom did not necessarily increase the pool of money allocated to provide the needed infrastructure. Shortages in electricity and water supplies have started to appear. The provision of highly subsidized water and energy puts pressure neither on the designers of the free housing to design sustainable buildings, nor on the users to invest in modifying their existing energy and water consumption patterns.

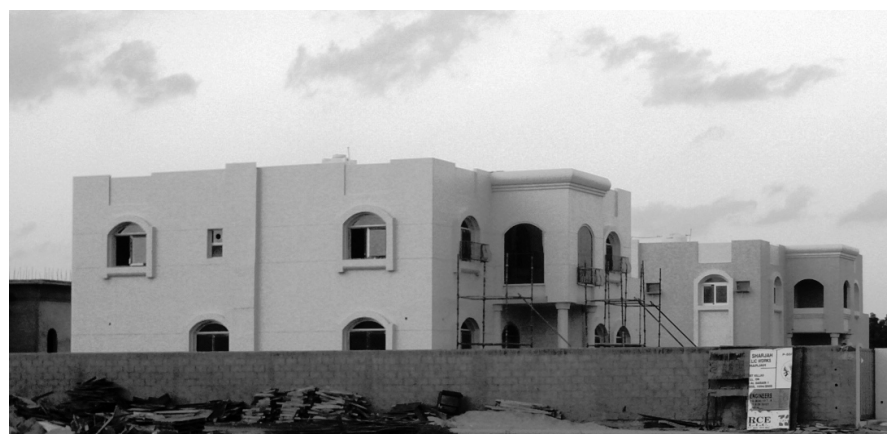

Figure 1: $\quad$ Sample of a free economic house. 
Increasing the price to cost level is seen as a destabilizing action and is not considered a viable option. The only feasible economic solution is clearly to make more effort to provide environmentally sensitive designs that consume less, and hopefully at the same time help users change their consumption patterns.

But the economic aspect is not the only force able to reduce energy and water consumption. In a region which has a hot climate and a very small industrial base, it is estimated that $70 \%$ of generated energy is used for the air conditioning of buildings. This energy is mainly generated by burning oil, thereby adding more $\mathrm{CO}_{2}$ in the atmosphere. In a desert region, the supply of water also requires the burning of oil to desalinate sea water. As there is hardly any agricultural activity, most desalinated water is used in buildings. The desalination process not only results in an increase in $\mathrm{Co}_{2}$ emissions but also affects the marine environment by depositing warm and highly saline water in the Arabian Gulf. As such, the need for environmentally sensitive buildings is increasingly recognized in the region. This is particularly the case with freely provided residences, as their design typically receives less attention and they are built in large numbers.

However, these buildings have additional problematic aspects. Designed by expatriates following standard international regulations that combine with a poor understanding of local culture, these residences do not adequately satisfy the needs of their occupants. As will be shown, privacy, the ability to receive guests with segregation of the genders, and the use of domestic servants, are among the important components in the cultural fabric of the region. The inability to address these components makes the users of these residences uncomfortable in their own homes, and gradually forces them to change their cherished traditions.

The paper shows an approach towards addressing some of the above mentioned challenges through a design approach that tries to integrate environmental and cultural requirements. The paper starts by investigating these requirements, and then demonstrates the proposed design approach in contrast to the traditional one. Finally, a sample design that is based on the proposed approach is shown and analyzed critically against both the cultural and environmental requirements. The author hopes that the proposed approach will trigger more discussion and investigation among academic and professional communities for the integration of cultural and environmental aspects of architectural design to achieve sustainability in both of these aspects.

\section{Investigating environmental requirements}

The Gulf Arab region is located on the western shore of the Arabian (Persian) Gulf. While its macro environment is that of a dry desert, the micro climate varies slightly due to factors such as the effect of the water in coastal areas and the effect of height on mountain areas. Therefore, we will assume a particular part of the region for which the reported study was developed. This part is the flat coastal area of the United Arab Emirates, which includes Abu Dhabi, Dubai, and Sharjah as its major cities. The two psychometric charts (Figures 2 and 3) 


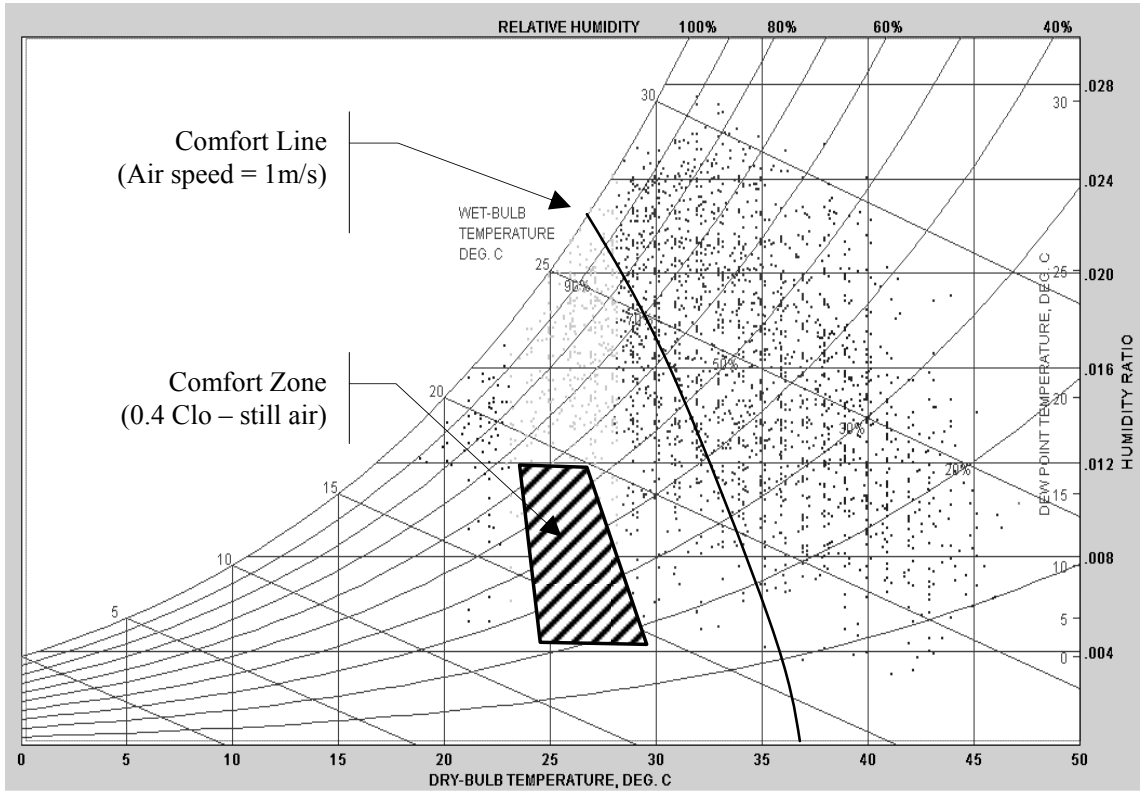

Figure 2: $\quad$ Abu Dhabi data during the hot season (June to October).

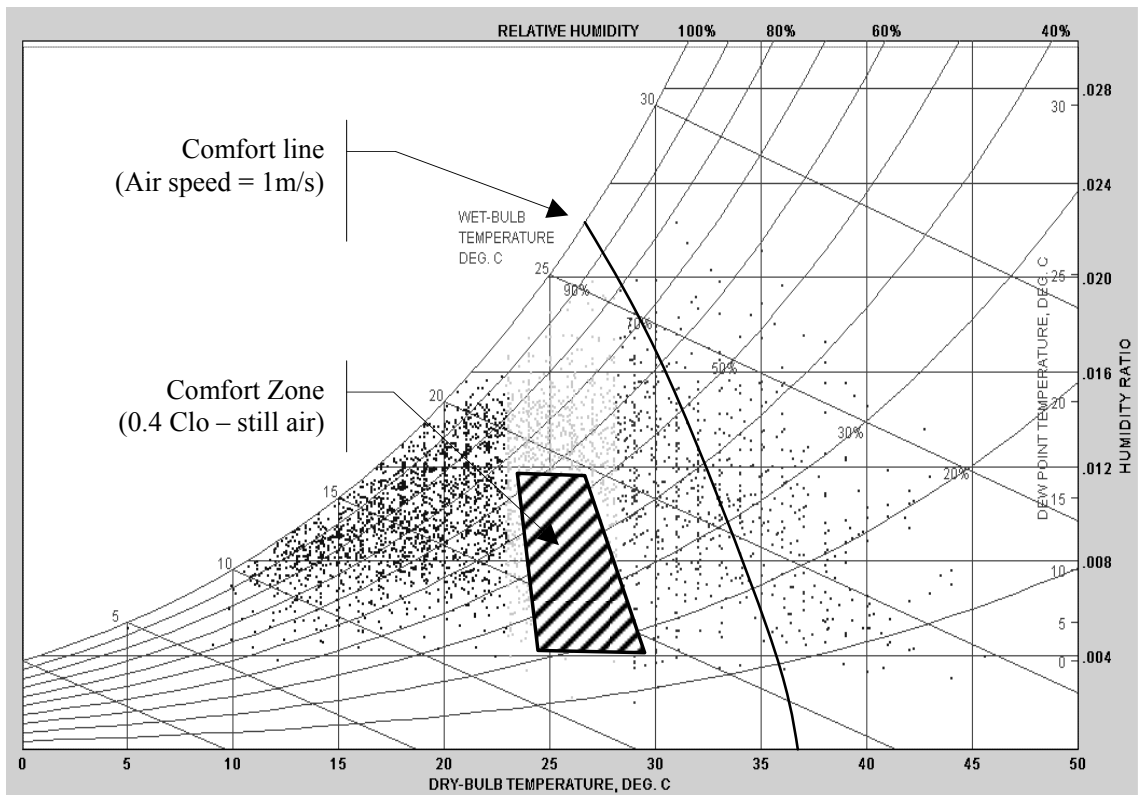

Figure 3: Abu Dhabi data during the temperate season (November to May). 
show the two main climatic seasons in this part of the world; a hot and humid climate for almost six months, tempered to a warm climate for the rest of the year. The climate data on the psychometric charts are for the city of Abu Dhabi and are shown using the software Climate Consultant [1]. Some of the preferred conditions for human thermal comfort are superimposed on the charts. The hatched zones indicate the comfort conditions for a typical sedentary person wearing light clothes when the air around the person is still. The curved line shows the possibility for the typical sedentary person to tolerate warmer and more humid conditions when the air speed moves up to $1 \mathrm{~m} / \mathrm{s}(3.6 \mathrm{~km} / \mathrm{h})$.

The data on the two charts shows the possibility of using air movement (natural ventilation) during most of the temperate season to achieve human thermal comfort and without the need for mechanical air conditioning. It also shows that air conditioning is required during most of the hot and humid season. Figure 4 shows the wind speed and direction for Abu Dhabi during the temperate season, using the software Climate Consultant. The data shows a wind speed of average $4 \mathrm{~m} / \mathrm{s}$ with dominant wind direction on the axis of North-West SouthEast. The shown wind speeds are measured at the airport at a typical height of $10 \mathrm{~m}$. With standard calculations, this wind speed can be translated to $2 \mathrm{~m} / \mathrm{s}$ at a height of 3 meters in a suburban area. Such a wind speed can be used for natural ventilation and can result in an average air velocity of $1 \mathrm{~m} / \mathrm{s}$ inside a building.

Other environmental data (not shown here) indicate abundance of solar radiation through most of the year. This indicates the need to provide shading to reduce solar heat gain. It also shows the potential of using solar energy to run air conditioning, provide domestic hot water, and generate some of the required electrical energy.
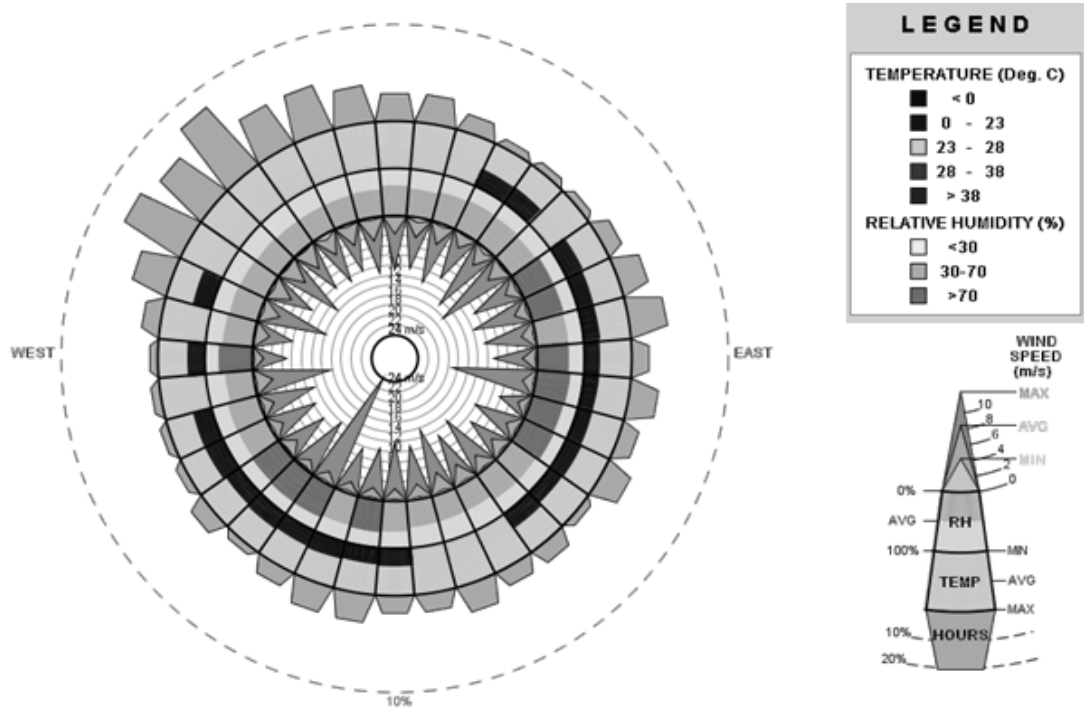

Figure 4: Abu Dhabi wind data during the temperate season (November to May). 


\section{Investigating cultural requirements}

The Arabian Gulf region has some particular cultural requirements that have been shaped by many factors, including religion, history and the natural environment. Anecdotal data collected by many discussions with the citizens (natives) of the region suggest the following cultural issues as being critical to the design of houses.

\subsection{Privacy}

Excluding immediate circle of family members, both males and females keep a very high level of privacy. If seen by outsiders, they need to wear clothes that cover almost all of the female body and most of the male body. As such, house design needs to ensure that people such as neighbors and passersby will not be able to see inside the house and view the residents wearing their relaxed house cloths. Failure to provide this privacy in current designs had resulted in residents being unable to use the house in comfort. It has also resulted in environmental implications as to be shown later.

\subsection{Guest segregation}

Historically, the natives to the region lived in isolated villages in the desert. Due to the harshness of the environment, travelers and guests were received with generosity to provide them with support. This generosity remains in the culture of the society and one or more spaces in a house are dedicated to the reception of guests. However, male guests sit with male family members while female guests sit with female family members in segregated spaces. Typically the space allocated to male guests is accessible from outside the house so that normal household activities are not interrupted. Female guests are typically invited into the living room inside the house if the male space is occupied. Food is typically served to the guests in these spaces; normally on the floor. Occasionally, some guests may need to sleep overnight. The guest bedroom needs to be isolated so it does not interrupt the privacy of the occupants.

\subsection{Domestic servants}

The relatively low cost of hiring domestic servants (maids and drivers) in the region makes their employment a standard practice. Domestic servants have their own rooms. The rooms of maids (typically female) should be apart from those of drivers (typically male). Preferably, domestic servants' rooms function independently of the rest of the house in the event that the owners are travelling and leaving behind some domestic servants.

\subsection{Kitchen extensions}

Because of the relatively large number of members in a family and the high frequency of guest visits, there is a need for large kitchens which are isolated 
from the rest of the house and have outdoor extensions. However, privacy needs to be ensured when cooking is performed outdoors.

\section{Design approaches}

The current approach for private residences in the region is locating a building in the middle of the allocated lot (Figure 5(a)). The author makes the case that this approach - which is common in many parts of the world - affects not only the social behavior of occupants but also their energy and water consumption, hence impacting both cultural and environmental sustainability in the region.

Because of the very high concern regarding privacy, residents of houses designed using the current approach are unable to use their outdoor spaces comfortably because their neighbors can see them; this means that they need to be wearing certain clothes. This factor impacts the quality of life, as residents cannot enjoy fresh air, nor gather outdoors, nor practice gardening, nor use a swimming pool. But the problem of privacy extends beyond this. Windows cannot be left open and need to be covered by curtains or have reflective glass that ensures privacy inside the house. The difficulty relating to the opening of windows makes cross ventilation an avoided solution, even when the weather would allow it. Hence, air conditioning is used continuously throughout the year to compensate for the lack of natural cooling. Daylight is also affected by the use of curtains and reflective glass. This results in greater use of artificial lighting. The impact of cultural issue on energy consumption needs to be recognized and integrated into the design.

In their attempts to achieve privacy, residents build walls around their houses (see Figure 1). This has become a standard requirement with the current approach. A typical wall will have reinforced concrete columns that are four meters apart. Each column has its own foundation and reinforced concrete tie beams that link these columns and carry masonry walls that extend between the columns. The materials used to build the walls can be as much as that needed to build the structure of the house itself. This results not only in cost increase but also in the consumption of materials that have large embedded energy and water.

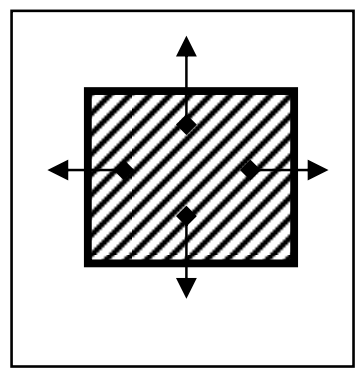

(a) Current approach

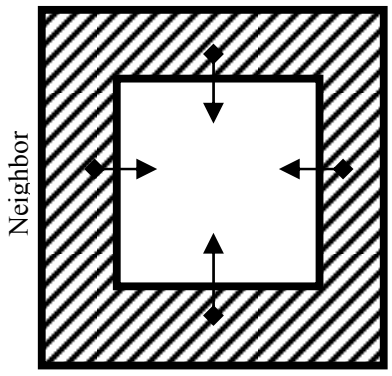

(b) Historical approach

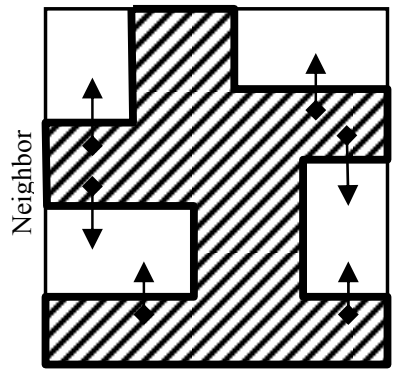

(c) Proposed approach

$\prec$ View Direction

Figure 5: Three design approaches for locating the residence mass on site. 


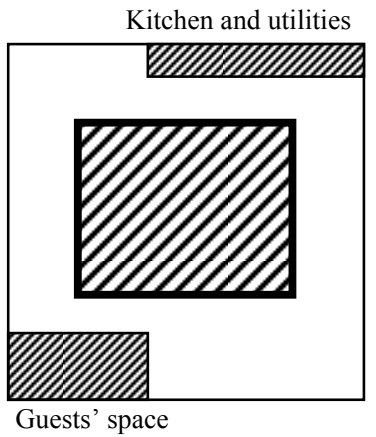

Figure 6: Typical location of the guests, kitchen, and utilities annexes.

The need for these walls can be evaded if the design approach integrates the cultural need for privacy.

While surrounding walls may help provide privacy from passers-by at ground level, it helps little in protecting upper floors, where bedrooms are typically located. Some occupants tend to use dense-leaved trees to secure some privacy for the upper floors. In a region where it hardly rains, this results in significant use of desalinated water to grow and maintain these trees.

The need to host guests in a space separate from the house is typically translated - with the current design approach - into an annex, as shown in Figure 6. Similarly, the kitchen and maids' rooms are treated as an annex. This lack of integration of these elements with the residence design contributes not only to the inability to use the outdoor spaces but also to the increase in energy consumption. These separate annexes are exposed to the sun with large roof areas relative to their volumes. This is particularly critical during the summer when the sun is almost perpendicular to the roofs.

A possible solution to the above-mentioned problems is to return to the historically used single courtyard approach (Figure 5(b)). This approach provides privacy through having most spaces looking inwards to the central courtyard. Guest rooms here need to look outwards so that guests do not violate the privacy of the courtyard. However, there are some issues with this approach. Cross ventilation is difficult to achieve for spaces that are sharing a wall with a neighbor. Circulation can be an issue, particularly during the summer. The single courtyard is typically used as the circulation space between the spaces around it. When air conditioning is used during the summer, residents need to circulate around the courtyard to move between the spaces. The courtyard needs to be closed in to enable the air conditioning of the circulation corridors around it. As some of the spaces have the courtyard as their only source of natural light, closing in the courtyard for the needs of air conditioning affects the natural light in these spaces. The historically used single courtyard approach does not fit well with current life styles where people of different ages and both genders are enjoying different types of activities (both indoors and outdoors) and where movement is very frequent between the different zones of the house. The approach put all these activities into a single space. 
The proposed design approach aims to avoid the problems of both approaches. As shown in Figure 5(c), it includes several courtyards within a mass that fills the whole site. The outdoor spaces are integrated into the mass and are not treated as leftovers. These external spaces serve both environmental and cultural roles in an integrated fashion.

From the environmental perspective, these outdoor spaces enable crossventilation through all the main indoor spaces during temperate weather. During summer, the proportion of these small courtyards (width to height) results in the shading of many of the external walls as well as the courtyard grounds. Limited numbers of native palm trees can provide further shading during times where the sun is at its peak angle in the sky.

From the cultural point of view, the windows of the surrounding spaces are oriented to open to the courtyards but in a parallel direction to the wall of the bordering neighbor. Minor treatment for these windows prevents neighbors from seeing each others' courtyard areas. Privacy can be easily secured and residences can enjoy using open spaces. The different courtyards can be assigned as private (seen and used by the family members only) and semi-private (seen and used by guests). Private ones can be further divided to accommodate a variety of activities by family members of different ages and genders.

\section{Proposed architectural design}

This section provides a sample design that uses the proposed approach in trying to integrate both cultural and environmental aspects. The design is developed under the guidance of the author by a student of the Architecture program at the American University of Sharjah. In the authors' opinion, the design addresses and integrates successfully most - but not all - the environmental and cultural concerns.

As shown in Figures 7 and 8, the design has two floors and includes four courtyards (C1 to $\mathrm{C} 4)$. The courtyards have relatively small dimensions in plan. Their widths relative to their heights provide adequate shading and hence minimize the sun's effect on the vertical elements of the envelope. However, the environmentally-useful small dimensions for the courtyards do not allow the violation of the residents' privacy due to the appropriate allocation of the functions of the spaces around these courtyards. At ground floor level, the outdoor space $(\mathrm{C} 2)$ is semi-private and is viewed by guests who occupy the guest room (5). The outdoor spaces (C1) and (C2) are typically private. However, if female guests are using the living spaces (9), they become semi-private. When the living spaces are used by guests, they do not violate the privacy of other parts of the residence. The swimming pool is located so that neighbors cannot view its users. The logia (8) location makes this possible (the plan is mirrored for the neighbor). The outdoor space (C3) is private and allows for an outdoor extension of the kitchen where residents can wear comfortable clothes while preparing food and are without fear of being viewed by neighbors, via minor window treatments to the proposed design. 


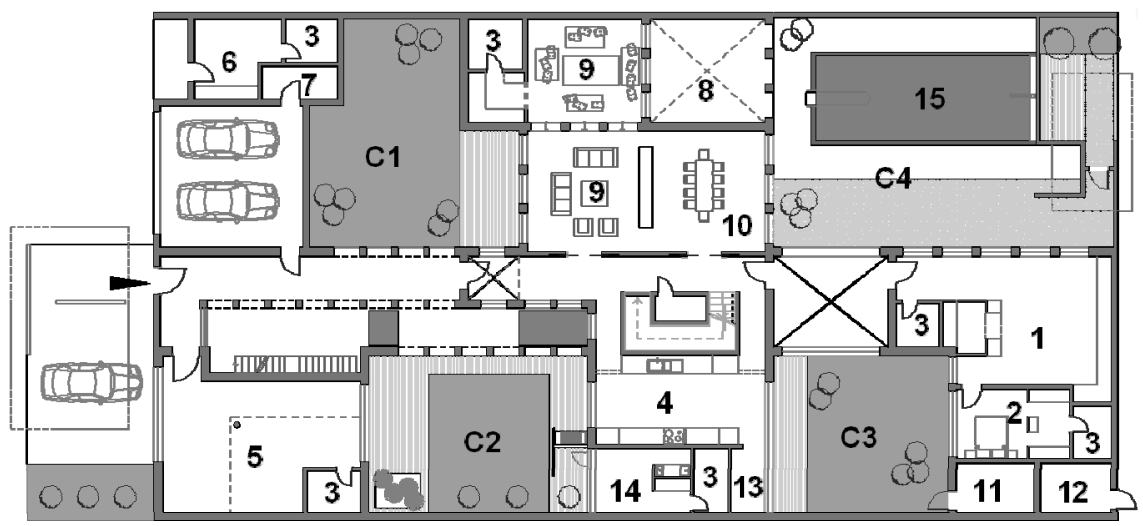

Figure 7: Ground floor plan of the proposed design.

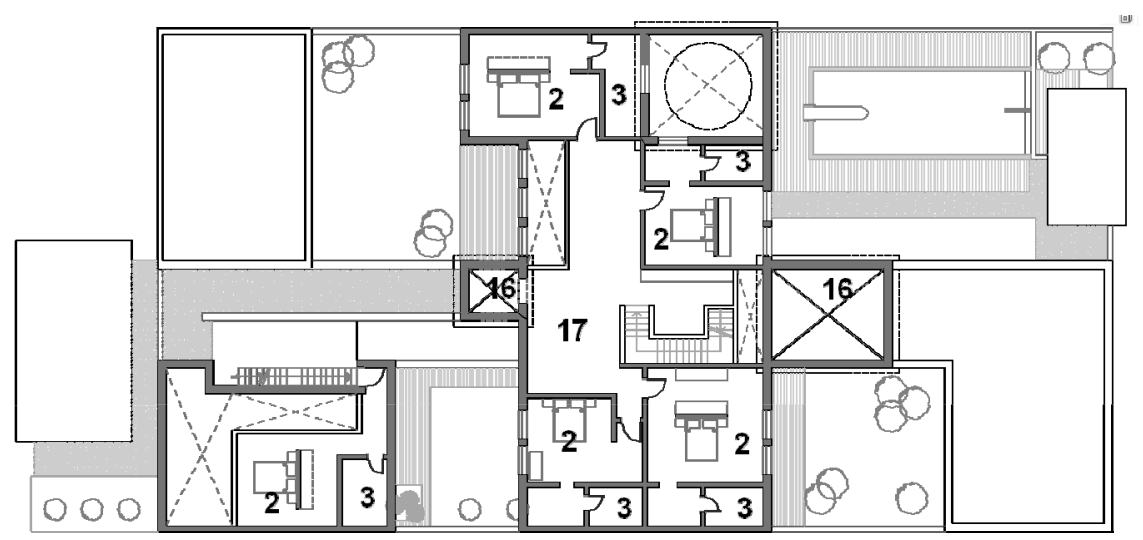

Figure 8: $\quad$ First floor plan of the proposed design.

Key to spaces:

1
2
3
4
5
6
7
8

$\begin{array}{ccc}\text { Family } & 9 & \text { Living } \\ \text { Bedroom } & 10 & \text { Dining } \\ \text { W.C. } & 11 & \text { Storage } \\ \text { Kitchen } & 12 & \text { Chiller } \\ \text { Majlis (guest room) } & 13 & \text { Laundry } \\ \text { Driver } & 14 & \text { Maid } \\ \text { Tools } & 15 & \text { Pool } \\ \text { Logia } & 16 & \text { Wind tower }\end{array}$

With the multi-courtyard approach, almost all spaces can be naturally ventilated through cross ventilation. All upper floor bedroom windows open on to the different courtyards, but are parallel to the border with the neighbor. Hence, privacy is provided for bedrooms as well as for the neighbors' courtyards 
(Note: the design assumes a front road and back pedestrian road. These roads make the distance to the back neighbors far enough to secure privacy. Minor window treatment is certainly needed)

All roofs are covered with vacuum tube solar collectors (not shown in the drawings) that heat water used to run an absorption chiller (12) that air conditions the residence during the hot season. Locating the solar collectors on the roof not only provides shading to the roof during the summer (environmental control issue) but also makes the roof non-usable and hence secures privacy for the neighbors (cultural issue).

Guest room (5), kitchen (4) and domestic servant rooms (14 and 6) are integrated into the design and are not annexes as in the current design approach. This makes these spaces less exposed to direct sun and hence reduces the amount of energy needed to cool them. Yet the guest room and guest bedroom represent a separate air conditioning zone, making it possible to save energy by switching off air conditioning when the zone is not in use.

As the proposed design is expected to be repeated, various orientations for the windows and courtyards are expected. Wind directions may not be facing the windows' orientation for some units. Two wind towers (16) are therefore provided to capture the wind from any direction and encourage natural ventilation during the temperate season. The location of the towers requires further study to make sure they can actually function as expected. The wind tower, however, has other cultural function as it emotionally links the natives of the region to their historical roots where wind towers were typically used.

\section{Conclusion}

This paper discussed a design approach and a design example that applies this approach for the integration of both cultural and environmental requirements to achieve multi-dimensional sustainability in affordable housing projects in the Gulf Arab region. The paper investigated these two types of requirements, explained the proposed design approach in contrast to the current and the historically used approaches, and critically analyzed a design example that used the proposed approach.

\section{Acknowledgement}

The author acknowledges Ms. Noura Omaira, a native of the United Arab Emirates and an alumnus of the American University of Sharjah for her effort in developing the design example. Ms. Omaira developed the design under the authors' guidance during her final design project as an architecture student at the university.

\section{Reference}

[1] Climate Consultant, UCLA Energy Design Tool Group, http://www.aud.ucla.edu/energy-design-tools 